\title{
Dopamine pathway induces emergence from anesthesia
}

Surgical procedures typically require patients to be placed under general anesthesia, which is usually well-managed and very safe. Emergence from anesthesia can have clinical complications, however, including delayed emergence, intraoperative decreases in oxygenation and emergence delirium, which can be associated with cognitive dysfunction and morbidity. There are no approved strategies for reversing the effects of general anesthesia in order to prevent these outcomes. Ken Solt, assistant professor of anesthesia at Harvard Medical School and Massachusetts General Hospital (Boston, MA), explained in a press release, "Clinically, emergence from general anesthesia is still a passive process during which we just wait for the drugs to wear off and the patient to wake up, a process that can take from a few minutes to an hour or longer. Finding a way to safely arouse patients from anesthesia... may reduce problems such as postoperative delirium and cognitive dysfunction."

Solt is leading an effort to find such a strategy. The neurological underpinnings of emergence from general anesthesia are not well understood, but results from recent studies have shown that certain drugs that activate arousal pathways in the brain can encourage emergence. For example, Solt's team previously reported that the stimulant methylphenidate, a drug used to treat attention deficit hyperactivity disorder, aroused rats from general anesthesia by activating dopamine pathways.

In Solt's new study, rats received electrodes implanted in one of two brain areas that contain dopamine-releasing neurons: the ventral tegmental area (VTA) or the substantia nigra. Application of a mild electrical current to the electrode stimulated dopamine release in the surrounding area. After the rats were anesthetized with one of two general anesthetic agents (isoflurane or propofol), an electrical current was applied to the implanted electrodes. Stimulation of the VTA, but not the substantia nigra, aroused the rats from anesthesia: they moved, lifted their heads and righted themselves (Anesthesiol. 121, 311-319; 2014). Electrical stimulation of the VTA also shifted the rats' brain activity to patterns associated with arousal. The effects of electrical

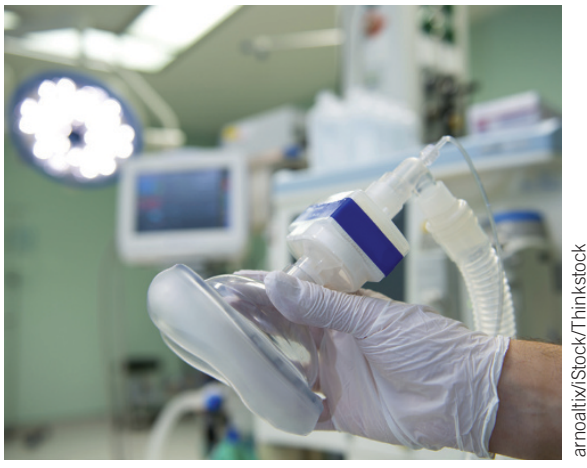

stimulation of the VTA in inducing arousal from anesthesia closely resembled the effects of pharmacological activation of dopamine receptors using methylphenidate. Overall, the results suggest that the arousal effect of methylphenidate is mediated by dopamine release specifically in the VTA. Activating the VTA at the end of a surgical procedure might therefore be used to induce emergence from anesthesia, stimulate consciousness and, according to Solt, "treat common postoperative emergence-related problems such as delirium and restore cognitive function."

Monica Harrington

\section{TINY FLY EARS INSPIRE A NEW HEARING DEVICE}

The fly Ormia ochracea can determine the direction and location of its prey, a chirping cricket, with remarkable accuracy for a creature so small. Larger animals typically use interaural time, or the difference in time between when a sound wave hits one ear versus the other, to determine the location of a sound. The ears of the fly are less than $2 \mathrm{~mm}$ apart, however, a distance 50 times smaller than the wavelength of the sound emitted by a cricket. Its hearing therefore relies upon a unique, sophisticated sound processing mechanism. This mechanism inspired researchers at the University of Texas in Austin to develop a prototype for a novel hearing device for use in humans.

Although the difference between a sound wave's time of arrival at each 0 . ochracea ear is a negligible 4 millionths of $1 \mathrm{~s}$, the phase of the sound wave shifts slightly between the first and second ears. The fly's ear contains a structure, only $1.5 \mathrm{~mm}$ in length, that detects this phase shift and uses two modes of vibration to amplify the interaural time and sound level differences so that the location of the sound can be determined.

The tiny prototype developed by Michael Kuntzman and Neal Hall mimics this structure and its properties. The device consists of a thin, flexible, silicone beam suspended by two pivots, resembling a miniature see-saw. Four springs wrap around the perimeter of the beam and connect to the left and right ends of the beam. In response to sound waves, the beam and spring structure has a 'rocking' mode of vibration, in which the beam rotates about the pivots, and a 'flapping' mode of vibration, in which both ends of the beam alternate their motions. The device also contains four sensing ports made of piezoelectric materials, which turn mechanical strain into electric signals. These ports measure the phase displacements that result from the sound wave (i.e., the flexing and rotation of the beam) to amplify the interaural time and sound level.

In experiments, the device replicated the fly's ability to detect sound direction and compute the angle of sound incidence via the piezoelectric sensing ports (Appl. Phys. Lett. 105, 033701; 2014). The researchers say that future versions of the device will only need two sensing ports and may include a third 'twisting' mode of vibration, which would make it possible to simultaneously sense gradients along both horizontal and vertical axes.

Kara Rosania 